\title{
A novel ESPI system for the analysis of static and oscillating displacements of microsystems
}

\author{
Kai-H. Lietzau' ${ }^{1}$, Carsten Stollfuß ${ }^{1}$, Andreas H. Foitzik ${ }^{1}$, Maria Richetta ${ }^{2}$ \\ ${ }^{1}$ University of Applied Science Wildau, Department of Engineering and Natural Sciences, 15745 \\ Wildau, Germany \\ ${ }^{2}$ University of Rome Tor Vergata, Department of Industrial Engineering, Via Orazio Raimondo, 18 - \\ 00173 Roma, Italy \\ kai-henning.lietzau@th-wildau.de, carsten.stollfuss@th-wildau.de,Andreas.foitzik@th-wildau.de, \\ richetta@uniroma2.it
}

\begin{abstract}
The presented research is focused on the adaption of the Electronic Speckle Pattern Interferometry for the analytical examination of displacements on so called MEMS. For doing so, a completely new experimental setup has been designed fitting the requirements of the optical measurement technique as well as the analyzed microsystems. Furthermore, it also has been possible to analyze the displacement of selected microsystems and consequently verifying this proof-of-concept.
\end{abstract}

Keywords: ESPI, interferometry, deformation, MEMS

\section{Introduction}

Since the invention of lasers in the 1960s, a number of physical methods of analysis and measurement have been established utilizing this unique light source. One of these methods is the Electronic Speckle Pattern Interferometry (ESPI). ESPI is a very capable optical technique which allows displaying deformations as well as oscillating displacements with a very high axial resolution. Commonly, this laser based technique is applied in the field of automotive engineering as well as in material-testing and is focused on the analysis of macroscopic specimens [1, 2]. One of the main topics is hereby the stress related mechanical behavior in terms of a reliability analysis.

Based on this technique, this work presents an experimental system which adapts this measuring principle for the analysis of deformation states on microsystems and thus heading for high lateral resolution, too. An initial prototype of the system has been designed and assembled including several functional modules. These include components of the damping system, the internal optics, environmental control units (temperature, air humidity and particle concentration), components of the beam path, a laser coupler, a mount for the microsystems and the necessary control unit. So far, such technological solutions are not yet commercially available or are only available in individual cases at an experimental state.

\section{Theory of Speckle Creation}

In general, the speckle phenomenon can occur under two different circumstances. Firstly, when an optical rough surface is illuminated with high coherent light. Optical rough means, that the selected wavelength is significant smaller compared to the height variations of the analyzed surface. In that case, each point of the surface scatters the light differently and, according to the Huygens-Fresnel principle, can be considered as the source of a new elementary wave (figure 1) [3]. Furthermore, these coherent wavelets have been differently modified concerning their amplitude and phase due to their individual origin. All these wavelets propagate away into space and interfere at a given point $\mathrm{P}(\mathrm{x}, \mathrm{y}, \mathrm{z})$ creating the speckle pattern. The result is not a homogenous illuminated surface, but rather a mixture of bright and dark spots variable in size and shape [4].

The second scenario for the creation of a speckle pattern is quite similar. Hereby a round glass is used as the diffuser similar to the optical rough surface in the first scenario. While the diffuser surface can be considered flat at the point of entrance, small local irregularities like curvature or spikes spread the transmitting wave into spherical wavelets at the exit point. These random inhomogeneity creates a phase modification of the emitting 
secondary waves. In both scenarios the created speckle are different from one another regarding their individual intensity, because the surface condition of their source or local area on the object is different from point to point. Therefore, each single speckle and each speckle pattern can be seen as a fingerprint of the corresponding local area on the object surface given at a certain state.

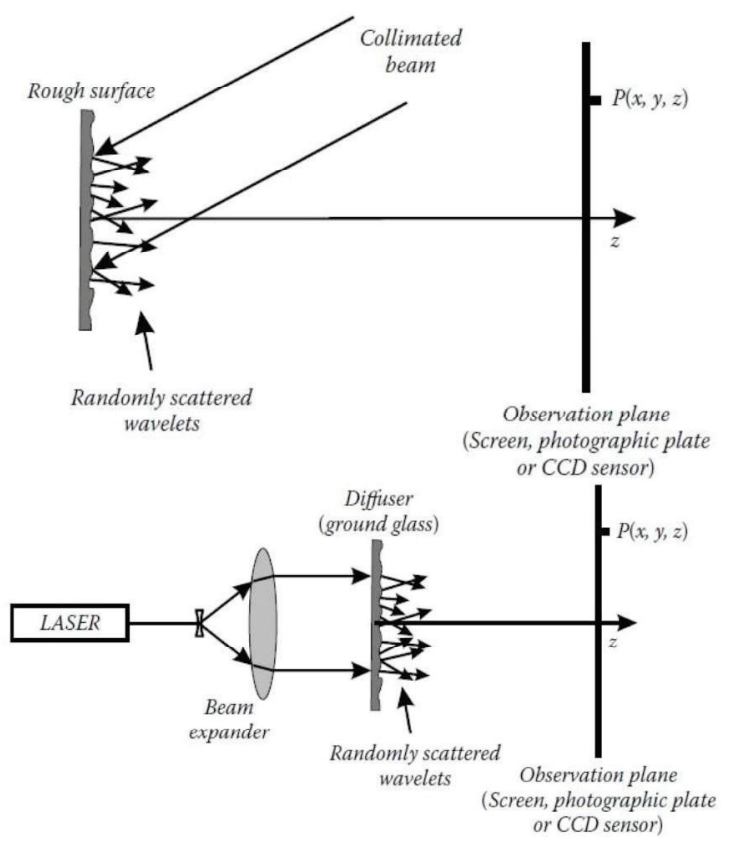

Fig. 1: Speckle creation, by scattering from an optical rough surface (above) and by propagation though an optical diffusor (below) [5]

Such speckle patterns are time-independent if (i) the position of the light source, (ii) the observation plane and (iii) the illuminated surface itself remain stationary. However, even in case of a slight movement the resulting speckle pattern will be altered. Conversely, if this is included into a proper experimental approach, this knowledge can be used to apply speckle patterns to characterize translations, rotations, and phase changes of a given rough or translucent object

In case of an actual measurement the scattered light from the object is first of all recombined with a reference beam. Therefore, the specific speckle pattern for the initial state is created. By utilizing a CCD camera this pattern can be stored electronically. After obtaining this first image, a static or oscillating deformation is induced onto the object and a second image is recorded. This second image contains all corresponding alterations between the initial and new state of the object. By subtracting these two images on a pixel by pixel basis it is possible to visualize the occurred displacement between both distinctive states.

Based on this approach it is possible to detect and to evaluate even smallest displacements for example such as vibrations. Most commonly ESPI is used to analyze the so called "out-of-plane" deformation of objects. This means, the displacement takes place alongside of the optical axis and either moves towards or away from the observation plane. Depending on the applied setup it is also possible to analyses the "in-plane" deformation [6]. Such displacements are vertical orientated to the optical axis.

As already pointed out before, ESPI is commonly used in the field of material engineering or associated engineering industries. Hereby, the provided insight of the deformation behavior enables further statements regarding the characteristics of the analyzed components (elasticity, plasticity, natural frequencies or even thermal expansion). Beside these advantages the ESPI has been limited to the analyzation of such macroscopic samples.

\section{Technological Approach}

Typically, samples which are analyzed utilizing ESPI setups are objects with a spatial dimensions in the centimeter and meter range. In order to measure smaller samples an adaptation of the original ESPI setup is needed. The development of such a microESPI is the topic of the presented research. By integrating additional magnifying optics and a complex environmental control system this new ESPI device should be capable to characterize displacements of micro-structured as well as micro-dimensioned samples. In total the requirements include:

$\begin{array}{ll}\text { - } & \text { Magnifying optics } \\ \text { - } & \text { Sample holders for microsystems } \\ \text { - } & \text { Housing of the complete setup } \\ \text { - } & \text { Vibration damping } \\ \text { - } & \text { Temperature control } \\ \text { - } & \text { Regulation of the humidity } \\ \text { - } & \text { Adaption of the control system } \\ & \text { Semi-automatic process control }\end{array}$

Fullfilling these requirements the micro-ESPI system should be able to investigate a large variety of micro-systems both qualitatively and quantitatively, aiming for stress-strain analysis and identifying manufacturing defects or features, respectively. 


\section{The experimental Setup}

Based on the individual requirements a initial concept of the micro-ESPI prototype has been realized to fit the requirements of the ESPI technique as well as the analyzed microsystems. The components include, a damping system, the required optics, a modular system for the control of enviromental factors such as the temperature or the humidity, a laser switch, an excitation systems for the analyzed MEMS and a control system. All components are furthermore mounte inside a supporting structure. The complete system is enclosed in a housing which also contains the necessary interfaces. Figure 2 contains a first concept in CAD as well as the finished sytem.

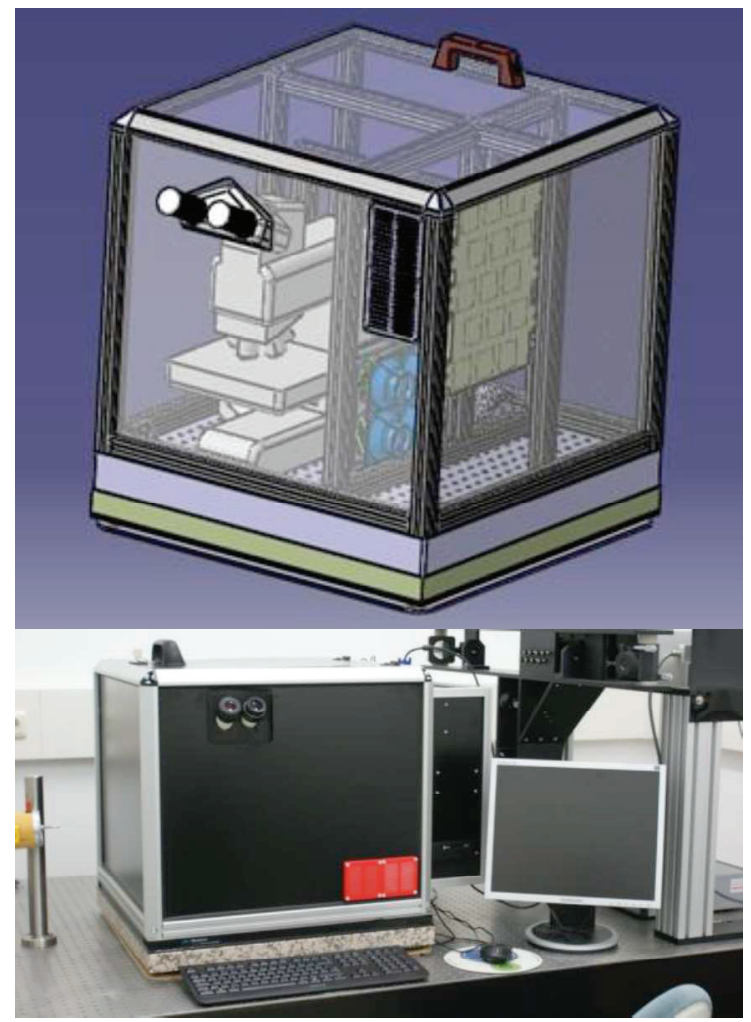

Fig. 2: Concept of the micro-ESPI in CAD (above) and the operational prototype (below)

\section{Damping System}

Chaotic external vibrations are one of the most important sources of noise. Consequently, the complete setup needed to be equipped with a damping system. As technological approach a passive system has been selected. Key element is a fully floating granite plate with the dimensions of $60 \times 60 \times 3 \mathrm{~cm}$ and a total mass of $40 \mathrm{~kg}$. By the principle of inertia, the natural frequency of the system has been therefore moved to the low-frequency range. Additionally, the granite plate has been equipped with TRI absorbers which filter in a wide range low-frequency vibrations.

\section{Internal Optics}

The optic of the micro-ESPI primarily contains a heavily modified Leica DM/LM microscope. Beside the magnifying optic this also provides a mount for the CCD camera and a basic sample holder. The structure is further complemented by two beam splitter and mirrors for the guidance of the optical beam path as well as additional optical filters. These are used for the regulation of the light intensity and for the filtering of possible interfering wavelengths. In case of an actual application, these filters are addressed directly by the control system for a better and reproducible tuning. Nevertheless, it is also possible to readjust them individually by hand.

In order to fit the requirements of the rather confined space all components needed to be as compact as possible without reducing the integrity of the beam path. Consequently, most of these parts have been completely created from scratch. Figure 3 displays as an example the CAD schematics as well as the manufactured components a mirror holder.

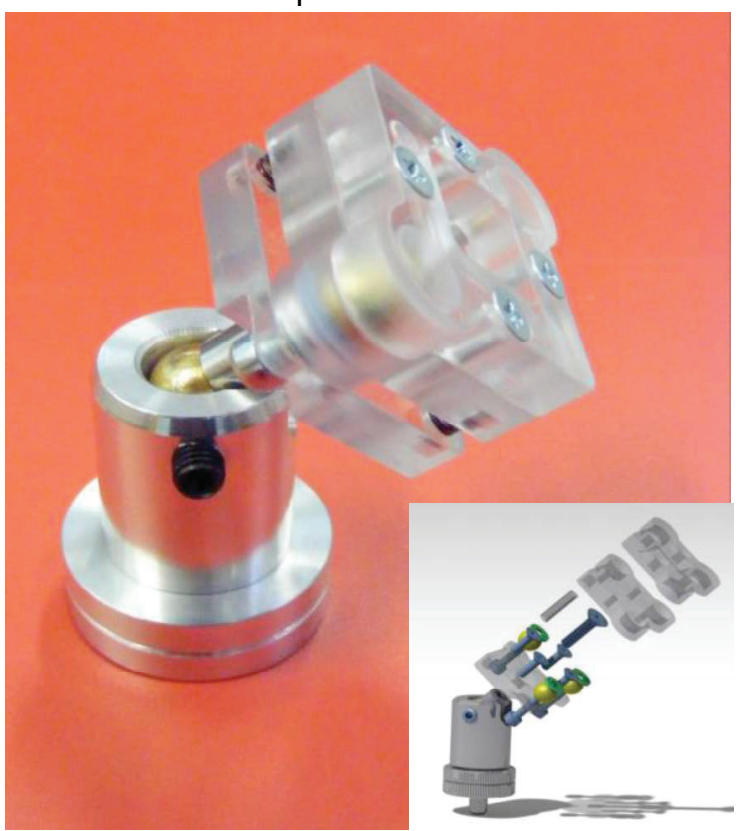

Fig. 3: Developed prototype of the mirror holder and the CAD concept (lower right)

\section{Environmental Control}

One of the most essential requirements of this research project has been the maintenance of the required measurement stability and therefore the reduction of external influences. Compared to macroscopic ESPI systems, this additionally includes fluctuations of the temperature, humidity and the particle load in 
the air. In order to control these factors a flow channel has been designed which provides a defined air flow into the measurement chamber. The channel contains several mount for Peltier elements which allows to adjust the temperature of the passing air flow. Figure 4 displays the flow channel and its different layers.

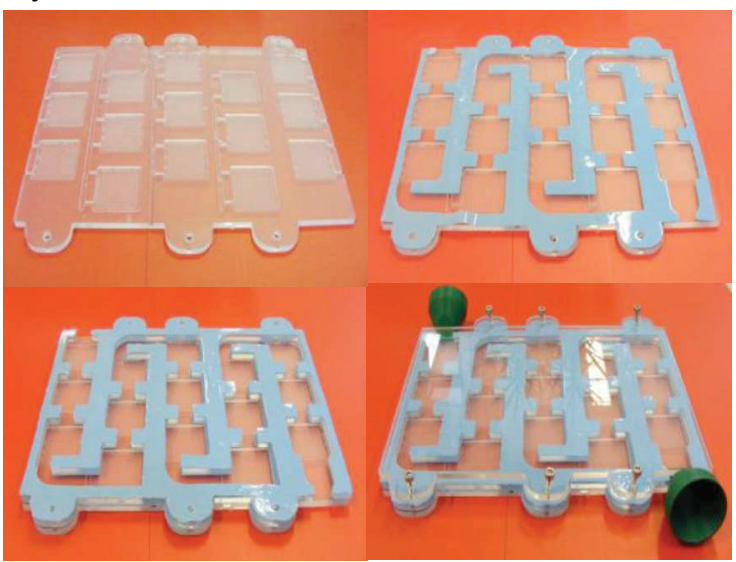

Fig. 4: Prototype of the cooling channel; ground plate (upper left), with silicon gasket (upper right), intermediate level (lower left) and top plate with funnels (lower right)

While in operation, the incoming air is first cooled (first 4 chambers) and then re-heated (last 4 chambers). This first cooling step dries the air and thus also ensures a precise humidity. The Peltier elements are triggered by the controlling logic and additional thermal sensors which are placed at the entry and exit of the channel as well as within the measurement chamber. The entire unit is ventilated via a particulate filter in combination with an active fan which pushes the filtered air into the system. Additional heat is dissipated through heat exchangers.

\section{Control System}

In order to provide the required reproducibility for the individual steps of operation, a complex control system has been integrated into the micro-ESPI. This system controls (i) the alignment of the mirror of the beam path in coordination with the laser source, (ii) the regulation of the environmental conditions in and around the measuring chamber (temperature and humidity) as well as (iii) the positioning of the test samples on the measuring table. Furthermore, the focusing and regulation of the laser light intensity are also partial regulated by this system.

Key element is a microcontroller (Arduino) which has been programmed and connected with the sensors as well as the electromechanical actuators. As shown in figure 5, the controller itself is mounted inside a PMMA housing. By an additional set of relays the microcontroller also handles the electrical and fluidic supply of the analyzed samples individually.

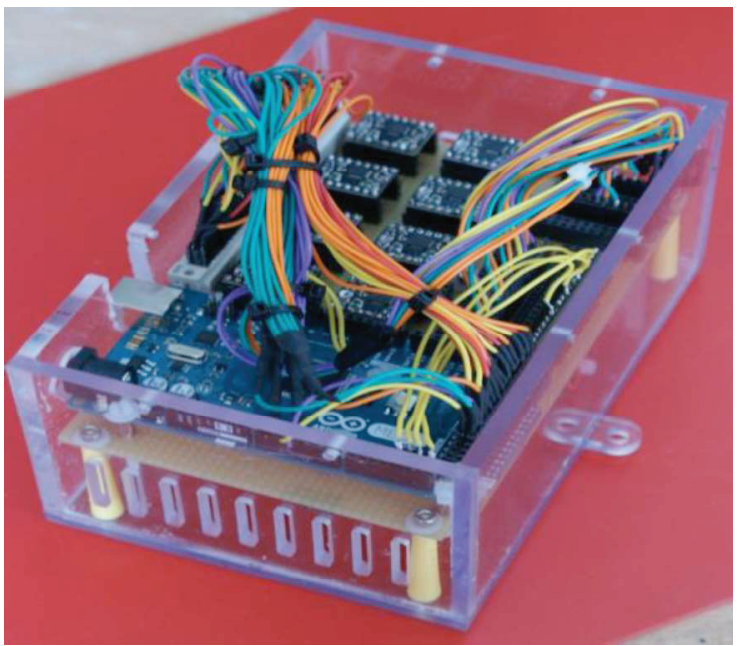

Fig. 5: In housed Arduino Mega 2560 and driver boards

Additionally, a PC is integrated into the setup to control the analysis software (Frames Plus), the microcontroller, to provide a basic data backup and as a user interface. The PC is furthermore thermally decoupled from the measuring range while any heat is dissipated through vents.

\section{Laser Switch}

Currently, the micro-ESPI contains a DPSS laser which emits laser light at a wavelength of $458 \mathrm{~nm}$ as light source. Nevertheless, it is possible to integrate different and even multiple light sources into the system. This is solved by a revolver switch. At first, the laser is guided via single-mode fibers, accordingly coupled in the revolver and then led into the micro-ESPI. The revolver contains of two components with three to one mounts for glass fibers. Both are rotatable against each other and thus enable the switching of the glass fibers and the laser.

\section{Excitation System}

In order to accommodate the microsystems, a universal holder has been designed which contains the sample in the measurement field and at the same time has the necessary connections to enable a functional test of the systems. The connection between the mount and the microsystem is carried out via terminal connections, but soldered connections are also possible. As mentioned before, the supply of the analyzed microsystem is case specific and individually controlled by the integrated microcontroller. 


\section{Experimental Results}

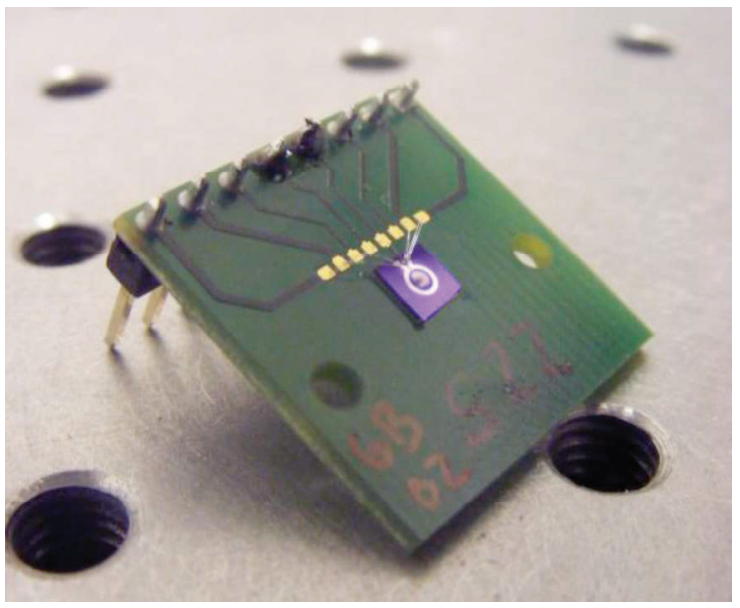

Fig. 6: Hydrogen sensor on circuit board

Several experiments have been carried out in order to provide reference values and to prove the initial presented concept of the micro-ESPI. These included simple tilting experiments as well as stress experiments for different microsystems.

One of the first sets of experiments have been focused on analyzing the thermal induced deformation behavior of a hydrogen sensor. This microsystem is displayed in figure 6 . As seen, the sensor contains a sensing area of palladium in the center as well as a surrounding platinum resistance heating. During the measurement, hydrogen will attach itself to the palladium surface which can be measured by a shift of the electric current. However, after the measurement is it necessary to remove this hydrogen. This is carried out by heating the sensing area by using the heating ring. However, if the thermal input is set to high, it can cause the detachment of the different material layers which would consequently result in a deterioration of the sensor.

During the experiments, the sensor has been exposed to thermal stress while monitoring different parts of its surface utilizing the ESPI technic. The following results have been obtained while focusing on the center of the sensing area. A first displacement of the surface has been detected with a power input of $5 \mathrm{~V}$ and $46.50 \mathrm{~mA}$ (figure 7). While increasing the input (figure 8), the deformation became circular shaped with a positive displacement at the outer edges. This could be reversed by simply reducing the power input. However, the shape became distorted and switched to one side of the measurement area (figure 9). It has been assumed, that at this point the material layers began to detach themselves from each other which consequently destroyed the sensor.

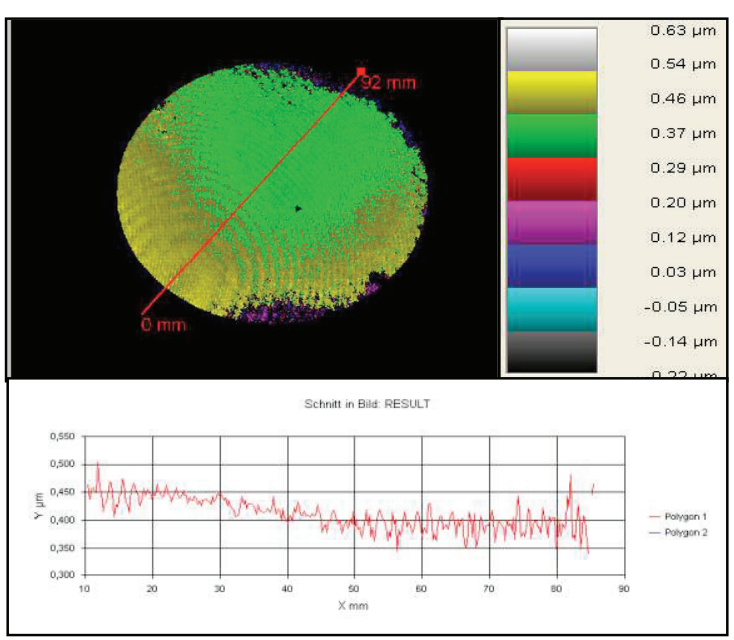

Fig. 7: Deformation of the sensor surface at an input of $5 \mathrm{~V}$ and $46.50 \mathrm{~mA}$

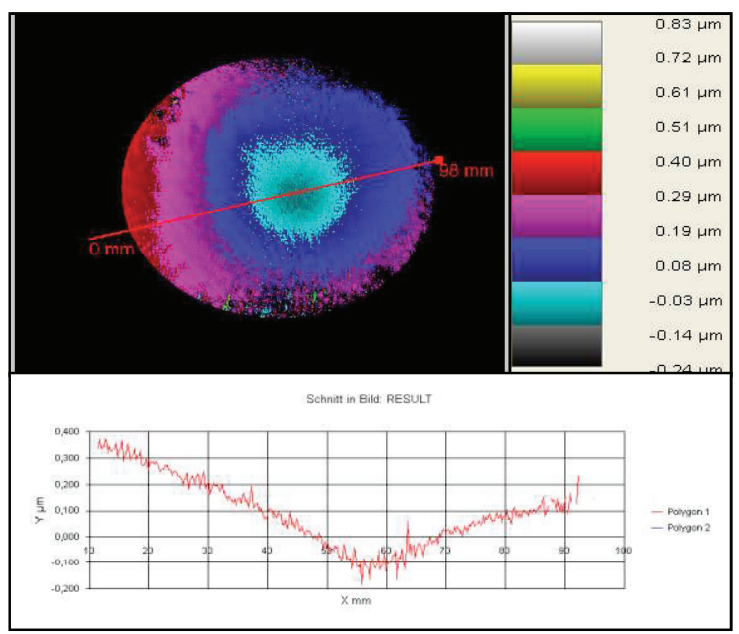

Fig. 8: $\quad$ Circular deformation at an input of $5 \mathrm{~V}$ and $46.50 \mathrm{~mA}$

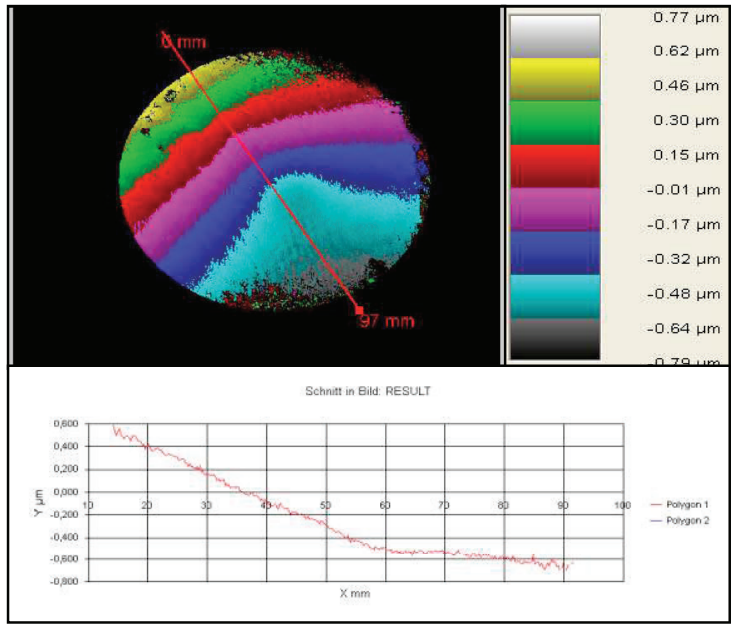

Fig. 9: Deformation at an input of $9 \mathrm{~V}$ and $170.00 \mathrm{~mA}$

In addition to the center of the sensing element, other area of the sensor such as the 
contact pads or parts of resistance heating have been investigated regarding their deformation behavior. In each of these experiments it has been possible to observe the thermally induced deformation of the sensor surface. With increase of the power input the outer region of the sensing surface continuously bulges radial upwards. However, a complete separation of the sensor surface has not been observed, but partial detachments.

\section{Summary \& Perspective}

Based on the initial concept it has been possible to adapt the ESPI for the deformation analysis of microsystems. Several components had to be redesigned in order to fit the requirements of the interferometric measurement method as well as the selected samples. This especially included the optical beam path as well as the modules for the control of external factors which strongly effects the reproducibility of the individual measurements.

Furthermore, first experiments have been carried out analyzing the influence of thermal stress onto a hydrogen sensor. During these experiments it has been possible to visualize the different stage of the displacement up to a partial separation of the sensor surface.

For the future it is intended to modify the sample mount and the measuring area for the processing of biological samples. This requires the additional sensors such as $\mathrm{O}_{2}$ and $\mathrm{CO}_{2}$ as well as a much more complex beam guidance.

\section{References}

[1] S. Yoshida, et al. "Electronic Speckle-Pattern Interferometry", Deformation and Fracture of Solid-State Materials - Field Theoretical Approach and Engineering Applications, Chapter 6, 165-171, Springer -Verlag, NewYork, (2015);

[2] W. Wang, C. Hwang, et al. „Experimental analysis of vibration characteristics of an edge cracked composite plate by espi method", International Journal of Fracture, 91:311-321, (1998)

[3] I. A. Razumovsky, et al. Interference-Optical Methods of Solid Mechanics. Foundations of Engineering Mechanics. Springer-Verlag, (2011)

[4] E. Hecht, et al. Optics, pages 602-603. Addison Wesley, 4.edition edition, (2002)

[5] N. G. Gaggiol and J. A. Pomarico, et al. "The Speckle Phenomenon", Dynamic Laser Speckle and Application, CRC Press, 21 - 35 pages, (2008);

[6] A.J. Moore, J.R. Tyrer, et al. "An electronic speckle pattern interferometer for complete in plane displacement measurement. Measurement Science and Technology, 1:1024-1030, (1990) 